\title{
Universiteit
}

Leiden

The Netherlands

\section{A force sensor for atomic point contacts}

Valkering, A.M.C.; Hulea, A.I.; Untiedt, C.; Babaei Gavan, K.; Oosterkamp, T.H.; Ruitenbeek, J.M. van; Babaei, Gavan K.

\section{Citation}

Valkering, A. M. C., Hulea, A. I., Untiedt, C., Babaei Gavan, K., Oosterkamp, T. H., \& Ruitenbeek, J. M. van. (2005). A force sensor for atomic point contacts. Review Of Scientific Instruments, 76, 103903. doi:10.1063/1.2084347

Version: $\quad$ Not Applicable (or Unknown)

License: $\quad$ Leiden University Non-exclusive license

Downloaded from: https://hdl.handle.net/1887/61323

Note: To cite this publication please use the final published version (if applicable). 


\title{
A force sensor for atomic point contacts
}

\author{
A. M. C. Valkering, A. I. Mares, C. Untiedt, ${ }^{\text {a) }}$ K. Babaei Gavan, ${ }^{\text {b) }}$ \\ T. H. Oosterkamp, and J. M. van Ruitenbeek \\ Kamerlingh Onnes Laboratorium, Universiteit Leiden, Postbus 9504, 2300 RA Leiden, The Netherlands
}

(Received 21 July 2005; accepted 30 August 2005; published online 5 October 2005)

\begin{abstract}
We have developed a sensor to study the mechanical stiffness of atomic-size contacts. It consists of a modification of the mechanically controllable break-junction technique, using a quartz tuning fork resonator as force sensor. We present first results of measurements of the force constants in gold atomic contacts. In the formation of chains of single-metal atoms, the folding in of individual atoms from the banks into the chain can be observed. This sensor allows one to measure forces in atomic contacts for a wide variety of metals, as illustrated with the first measurements on platinum. (C) 2005 American Institute of Physics. [DOI: 10.1063/1.2084347]
\end{abstract}

\section{INTRODUCTION}

Studying the electrical properties of the smallest possible conductor, a single atom, has become feasible in the last decade. ${ }^{1}$ It was shown that the conductance can be described with Landauer's formula $G=G_{0} \Sigma_{n} T_{n}$, where $G_{0}$ is the quantum unit of conductance, equal to $2 e^{2} / h$, and $T_{n}$ is the transmission of the $n$th channel. The number of open channels per atom is related to the valence of the atom, and varies from 1 for $s$ metals to 5 for $d$ metals. ${ }^{2,3}$

Two basic techniques are applied to create a single-atom contact, namely scanning tunneling microscopy (STM) (Ref. 4) and the mechanically controllable break-junction technique (MCBJ). ${ }^{5}$ Both techniques make a single-atom contact through the same principle: by stretching the contact, the diameter of the contact area decreases until only a few, and ultimately only one, atoms are in the cross section. Measured traces of the conductance as a function of the stretching reflect the reconfiguration of the atoms, and thus the number of available channels for conduction.

Although the electrical properties of atomic-sized contacts have been studied extensively, the mechanical properties have not yet received much attention. It can be expected that the two are quite closely related, as can be seen already from the example mentioned in the previous paragraph. Phenomena which have been mainly observed indirectly through conductance, such as the formation of atomic chains ${ }^{6}$ or the shell effect in metallic nanowires, ${ }^{7}$ could potentially be studied in far more detail via their mechanical properties.

The number of studies of mechanical properties of atomic contacts is limited; ${ }^{8-13}$ the most detailed ones concern measurements of the force through a combination of STM and atomic force microscopy (AFM) by Rubio et al. ${ }^{9,13}$ However, the number of metals that can be studied with this technique is small, because the lack of UHV surface preparation facilities limits the studies mainly to gold. Further-

\footnotetext{
${ }^{a}$ Present address: Departamento de Fisica Aplicada, Facultad de Ciencias (Fase II), Universidad de Alicante, Spain.

${ }^{b)}$ Present address: Molecular Electronics and Devices, Kavli Institute of NanoScience, TU Delft, Lorentzweg 1, 2628 CJ Delft, The Netherlands.
}

more, the stiffness of the cantilevers should be much larger than the force constant of the atomic contact, which compromises the sensitivity. For soft cantilevers the elastic energy built up would otherwise be suddenly released at a jump in the atomic configurations, giving rise to a large jump in distance. $^{14}$

For these reasons, we have decided to design a sensor capable of measuring mechanical properties. We use a quartz tuning fork resonator in combination with the mechanical break-junction technique. Tuning fork resonators have recently been introduced in different scanning probe microscopy techniques, such as AFM, ${ }^{15-18}$ magnetic force microscopy (MFM) ${ }^{19}$ and scanning near-field optical microscopy (SNOM) ${ }^{20,21}$ Compared to the standard cantilevers that are used in AFM, the tuning fork has a high spring constant $\left(k \approx 10^{4} \mathrm{~N} / \mathrm{m}\right)$, which in combination with a high quality factor allows high sensitivities at small oscillation amplitudes. ${ }^{22}$ For the various methods, different probes can be glued on the tuning fork, e.g., tips for AFM and STM, or an optical fiber for an SNOM. For our experiment, we glue a thin metal wire onto one of the prongs of the tuning fork. This can be done for a wide range of metals, thus giving us large flexibility in metals that can be studied. A similar force sensor and MCBJ combination has recently been developed independently by Rubio et al. ${ }^{12}$ With this sensor we can measure the stiffness, or force constant $k$, equal to the derivative of the force with respect to the relative displacement of the wire ends, $\partial F / \partial x$.

In the next section we will discuss the design of the sensor in more detail. After that, characterization measurements for different parameters of the tuning fork will be shown. In the last two sections we will present the first measurements that we have done with the tuning fork, for gold, to compare with earlier measurements, and for platinum, which demonstrate that experiments are possible for metals other than gold.

\section{DESIGN OF THE TUNING FORK SENSOR}

A schematic drawing of the tuning fork incorporated in an MCBJ is shown in Fig. 1. The prongs of the tuning fork 
a)

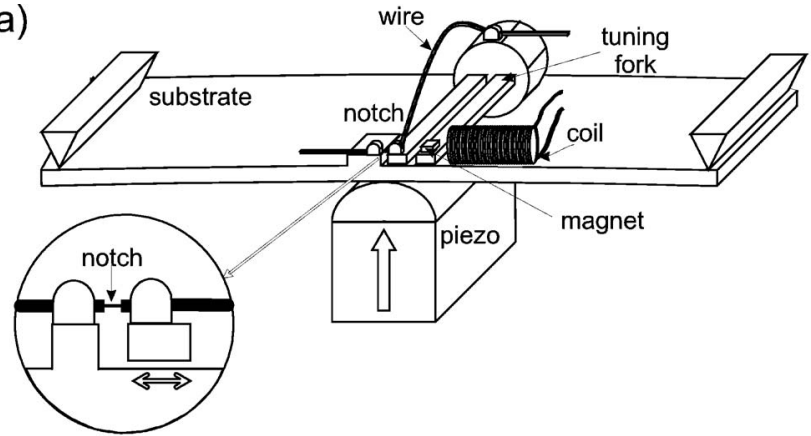

b)

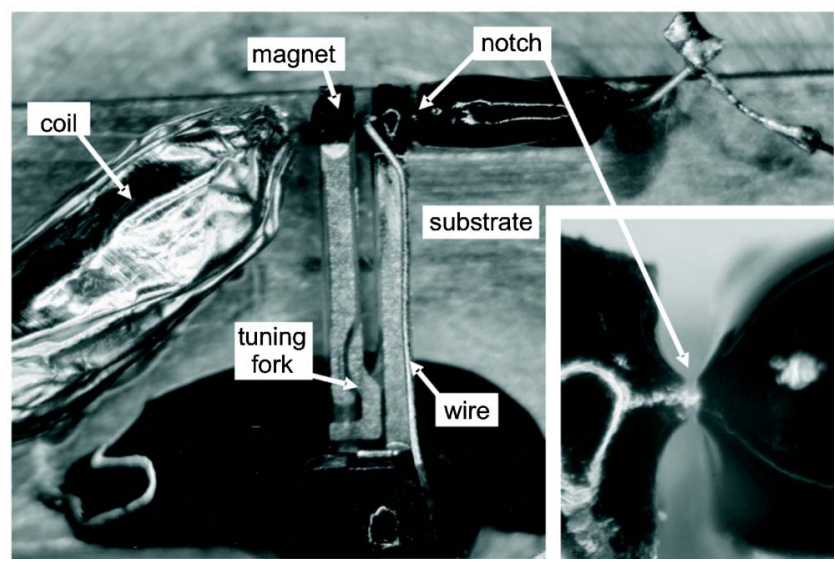

FIG. 1. (a) Sketch and (b) picture of the tuning fork resonator MCBJ. Inset in (b): Picture of the notch. The substrate is bent with the piezo element, causing stretching (and finally breaking) of the wire at the notch. The tuning fork resonance frequency is sensitive to the force applied on the wire. The coil and the magnet are used to drive the tuning fork

are $0.3 \mathrm{~mm}$ wide, $0.4 \mathrm{~mm}$ thick, and about $3 \mathrm{~mm}$ long. Its resonance frequency is $32768 \mathrm{~Hz}$. The tuning forks, as bought commercially, ${ }^{23}$ are mounted in a glass sealing with a metal cap on it. They have electrodes already attached, allowing the resonance to be detected through the piezoelectric effect of the quartz material. We remove the main part of the cap, leaving only a little ring around the base. This part is then soldered onto a substrate such that the two prongs are hovering just above it, with the preferred oscillation mode (the antiflexure mode) parallel to the substrate. Next to the tuning fork a little metal block is mounted on the substrate, such that the top of the block and the top of the tuning fork are at the same height. A $100 \mu \mathrm{m}$ thick wire with a notch (diameter $\approx 30 \mu \mathrm{m}$ ) is then glued on both one prong and the block, with the notch in between the prong and the block. The wire is stretched (and thus the notch diameter thinned down) by bending the substrate. For this the substrate is mounted in an insert in a three-point bending configuration, with the middle point beneath the notch. The bending of the substrate is done in vacuum at liquid helium temperatures to keep the wire fracture surface and atomic contact clean of adsorbates, thus circumventing the problem of clean surface preparation. The insert is pumped down to $10^{-6} \mathrm{mbar}$ at room temperature; at $4 \mathrm{~K}$ the pressure will be orders of magnitude lower, due to cryogenic pumping of the walls of the container. Since the force constant $k$ of a $30 \mu \mathrm{m}$ notch is about two orders of magnitude larger than $k$ of the tuning fork, in the beginning most of the stretching will be taken up by the tuning fork. As the notch becomes thinner, and thus $k$ lower, the notch will take over the bigger part of the stretching. We estimate, on the basis of bulk material properties, that the maximum displacement of the tuning fork during breaking of the notch is $0.1 \mathrm{~mm}$. In practice, for strong materials it is essential to cut a sufficiently deep notch to prevent the tuning fork from breaking. It helps to weaken the notch by bending the wire a few times before mounting it.

\section{DRIVING THE TUNING FORK}

Measurements are done at low temperatures, $4.2 \mathrm{~K}$, to keep the wire surface clean after breaking and to reduce vibrations. A consequence of the low temperatures is that the thermal vibrations of the tuning fork are small. For our tuning fork with a $k$ of $\sim 10^{4} \mathrm{~N} / \mathrm{m}$, the root-mean-square (rms) thermal amplitude is $\sim 10 \mathrm{fm}$, resulting in a piezoelectric current of order $10 \mathrm{fA}$. This current is below the noise level of the amplifier. For this reason, it is necessary to apply an external drive to the tuning fork. This can be done either directly using the piezoelectric effect or indirectly, shaking the tuning fork mechanically. We have tested both methods.

Applying an ac voltage to the electrodes of the tuning fork leads to a mechanical motion of the prongs due to the piezoelectric property of quartz. However, the applied ac voltage gives rise to a current through stray capacitances parallel to the tuning fork which makes the resonance curve strongly antisymmetric. Moreover, the phase becomes a nonmonotonic function of frequency, making it difficult to use a phase-locked loop (PLL). The parallel capacitance can be compensated for by a simple electronic circuit (see, e.g., Ref. 24). We have to realize, however, that the compensation needs to be quite accurate. The capacitance to be compensated is of the order of $100 \mathrm{pF}$ (due to $1.5 \mathrm{~m}$ of cables in the insert), whereas an uncompensated capacitance of $0.1 \mathrm{pF}$ leaves only a $0.5 \%$ window around the resonance frequency where a PLL can be used.

For the mechanical driving we prefer using a magnetic method. We have glued a small magnet on the top of the free prong (the one that is not used for the wire). Next to the tuning fork we glue a small copper wire wound coil, with dimensions approximately $8 \mathrm{~mm}$ length and $2.5 \mathrm{~mm}$ diameter with an iron core of $1 \mathrm{~mm}$, at a distance of approximately $0.5 \mathrm{~mm}$ away from the tuning fork. A magnetic field is induced in the coil by sending an ac current through it at the resonance frequency of the tuning fork. The stray field outside the coil moves the magnet on the prong and thus excites the tuning fork. The coil is wrapped in aluminum foil to screen the coil electrically.

In both methods the driving voltage amplitude is constant, rather than the observed signal amplitude. This is not very usual in a phase-locked loop system, but it does offer the possibility to directly measure the dissipation in the system from the observed amplitude.

The phase-locked loop was realized with a homemade system, consisting of a lock-in amplifier controlled by a PC via an IEEE (or GPIB) interface. The internal oscillator of the lock-in is used to drive the tuning fork. The phase shift is measured with a LABVIEW ${ }^{25}$ program. From this phase shift 
the resonance frequency is calculated and sent to the lock-in amplifier. This process is repeated until the phase shift is within a preset accuracy (usually $0.5 \mathrm{deg}$ ).

Once the change in resonance frequency $\Delta \omega$ is known, the change in force constant $\Delta k$ can be calculated

$$
\Delta k=\frac{2 k}{\omega} \Delta \omega,
$$

with $k$ and $\omega$ the force constant and resonance frequency of the sensor, respectively. It is clear that only changes in the force constant can be measured, and we have to choose a reference point. Usually this reference point is set to zero at the frequency and force constant for a broken contact.

\section{CALIBRATION OF THE OSCILLATION AMPLITUDE}

Although the amplitude of the tuning fork's oscillation is not a direct parameter in our experiments, it is still important to know it. The tuning fork oscillates in the direction of the axis of the atomic contact. During the measurement the contact should not be significantly modified, i.e., the amplitude should be small enough to prevent atomic reconfigurations. If we keep the amplitude below a few picometers, which means order $1 \%$ of the size of an atom, this condition is satisfied.

The standard method for measuring the amplitude is an optical one, using interferometry. ${ }^{18,26}$ We can, however, make use of the notched wire attached to the tuning fork to make the calibration. For sufficiently large separation of the wire ends, the tunneling current $I_{t}$ between two metal surfaces depends exponentially on the distance $d$ between them

$$
I_{t}=K V \exp \left(-2 d \sqrt{2 m \phi / \hbar^{2}}\right),
$$

where $V$ is the bias voltage applied across the contact, $m$ is the mass of the electron, $K$ is a constant dependent on the geometry of the electrodes and the density of states at the Fermi level, and $\phi$ is the work function of the metal. Due to the oscillation of the tuning fork with amplitude $A$ and frequency $\omega$, this current will have an additional oscillatory component, such that the total current becomes

$$
\begin{aligned}
I_{t} & =K V \exp \left(-2\left[d_{0}+A \sin \omega t\right] \sqrt{2 m \phi / \hbar^{2}}\right) \\
& =I_{\mathrm{dc}} \exp \left(-2 A \sin (\omega t) \cdot \sqrt{2 m \phi / \hbar^{2}}\right),
\end{aligned}
$$

where $I_{\mathrm{dc}}$ is the (dc) current with the two surfaces at a distance $d_{0}$, the equilibrium position of the tuning fork. For small amplitudes, $A \ll \hbar / 2 \sqrt{2 m \phi}$, the current becomes linear with the vibration amplitude

$$
I_{t} / I_{\mathrm{dc}} \approx 1-2 A \sqrt{2 m \phi / \hbar^{2}} \sin \omega t .
$$

When the current is measured with a current amplifier, the frequency-dependent transfer function of the amplifier has to be taken into account. With a gain resistance $R_{g}$ and parallel stray capacitance $C_{g}$, the total gain impedance $Z_{g}$ becomes

$$
Z_{g}=\frac{R_{g}}{\sqrt{1+\left(\omega R_{g} C_{g}\right)^{2}}}
$$

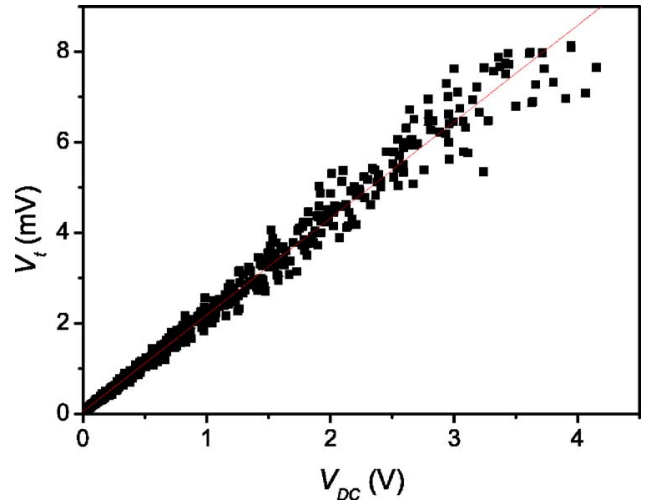

FIG. 2. Calibration of the oscillation amplitude of the tuning fork. The voltage at the current amplifier due to the oscillating tuning fork as a function of the voltage due to the dc tunnel current is plotted. The oscillation amplitude can be calculated from the slope.

With this, the expression for the ratio of the measured ac voltage amplitude over the measured dc voltage $V_{t} / V_{\mathrm{dc}}$ becomes

$$
\frac{V_{t}}{V_{\mathrm{dc}}}=\frac{2 A}{\hbar} \sqrt{\frac{2 m \phi}{1+\left(\omega R_{g} C_{g}\right)^{2}}} .
$$

Figure 2 shows a typical example of a calibration measurement. From the slope of the plot the amplitude $A$ can be calculated. With values of $R_{g}=500 \mathrm{M} \Omega, C_{g}=0.2 \mathrm{pF}$, $\phi=5.5 \mathrm{eV}$ (gold), and a frequency of $26 \mathrm{kHz}$, the amplitude is equal to $2 \mathrm{pm}$, the desired value.

Equation (2) can also be used to calibrate the displacement between the electrodes as a function of the voltage $V_{\text {piezo }}$ applied to the piezo element that is used to bend the substrate. $^{27}$

\section{EXPERIMENTS ON GOLD}

Figure 3 shows a typical trace for a gold wire with the change in force constant $\Delta k$ measured simultaneously with the conductance, as a function of the piezo voltage, which is linear to the displacement of the electrodes. As discussed in Sec. III we can only measure $\Delta k$ with respect to the total $k$ at the reference point. Here, we chose as a (natural) reference point the broken contact at large separation. The force con-

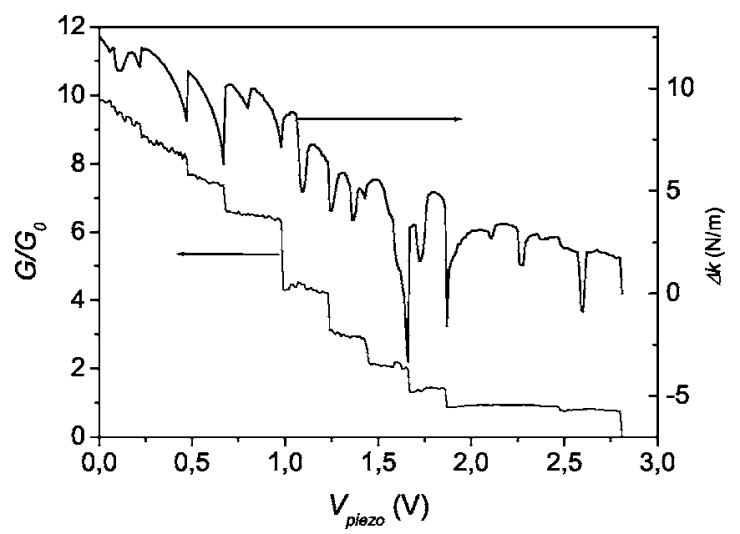

FIG. 3. Plot of conductance and changes in the force constant for gold as a function of the piezo voltage, which is linear to the elongation of the contact. 
stant decreases with decreasing conductance, which is expected since a smaller cross section will simultaneously be seen as a decrease in the conductance and in the stiffness. Sudden changes in the conductance are accompanied by an abrupt change in the force constant, pointing to a reconfiguration of the atoms at this point. But, also when only small changes in the conductance can be observed, for example at $V_{\text {piezo }}=0.8 \mathrm{~V}$, an abrupt change in the force constant is visible, indicating also that reconfiguration of atoms can take place, which hardly influences the conductance. Earlier measurements using conductance fluctuations ${ }^{28}$ and thermopower ${ }^{29}$ as a probing tool have confirmed that changes in the atomic configuration often occur far from the narrowest point of the junction.

Note also that the force constant is at some points negative with respect to the broken contact (for example, around $V_{\text {piezo }}=1.8 \mathrm{~V}$ ). It implies that at these points the pulling force exerted by the atomic contact on the prong of the tuning fork decreases with further stretching of the contact. The contact becomes soft at these instances, and finally results in atomic rearrangements.

The absolute value of the change $\Delta k$ between a one-atom contact and a broken contact is in this experiment of order $2 \mathrm{~N} / \mathrm{m}$. From the force measurements reported in the literature, ${ }^{9,10,13}$ we can calculate the $\Delta k$ by taking the slope of the force-distance curves. Typical values for a one-atom contact are $\Delta k=4 \mathrm{~N} / \mathrm{m}$ (Ref. 9) and $\Delta k=2-10 \mathrm{~N} / \mathrm{m} .{ }^{10}$ For a chain of atoms the lower value of $\Delta k$ during breaking is also $4 \mathrm{~N} / \mathrm{m} .{ }^{13}$ All measured values are in reasonable agreement. We have to keep in mind that the $\Delta k$ values calculated from the slope of the force-distance curves are an average over a distance where there is only elastic deformation between structural rearrangements. In our experiments we measure the $\Delta k$ directly, and it is clear from Fig. 3 that $\Delta k$ is not constant between the structural rearrangements. Rubio et $a l .{ }^{12}$ have also directly measured the force constant. The numerical value for a one-atom contact can only be estimated, since only the frequency change is given for this particular experiment. From comparison with other measurements in the article, we estimate a $\Delta k$ of $4 \mathrm{~N} / \mathrm{m}$.

The formation of an atomic chain can also be studied with the force sensor, as can already be seen in Fig. 3. A further example is given in Fig. 4 , where $\Delta k$ is shown on the conductance plateau at $G=1 G_{0}$. The length of the plateau already indicates that a chain of atoms is formed, as was shown by Yanson et al. ${ }^{6}$ In this case the reference point was set at the point where the conductance jumps to $1 G_{0}$. We can see a gradual increase as a function of distance over a length of approximately $0.25 \mathrm{~nm}$ until a sudden relaxation (dip in the force constant) takes place. At this point a new atom comes into the chain from the "banks," the parts of the wire near the contact. The strain that was built up when pulling on the chain is released. This agrees very well with the distance between atoms in the chain, as obtained from a length histogram. ${ }^{27}$ This pattern repeats itself until the chain breaks. In this case the breaking takes place after $0.85 \mathrm{~nm}$, when three atoms have entered the chain. The jumps of $\Delta k$ are of order $0.15 \mathrm{~N} / \mathrm{m}$, which is an order of magnitude less than the values for breaking a one-atom contact. When an atom

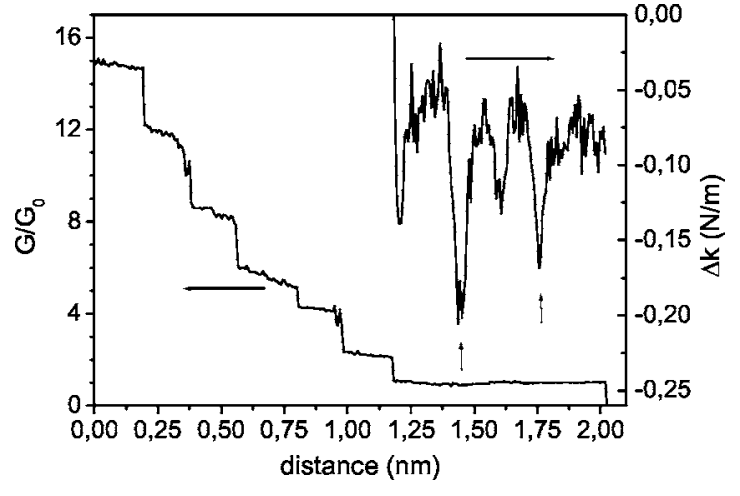

FIG. 4. Measurement of the force constant at a conductance plateau for gold. Dips in the force constant where a atom comes into the chain are indicated by arrows. The reference point was set at the point where the conductance jumps to $1 G_{0}$.

moves from the bank into the chain, bonds between the moving atom and the atoms in the bank are broken; therefore, $\Delta k$ may be expected to be of the same order. However, with the force sensor we measure not only the force constant of the chain, but also of the banks. In fact, the measured $k$ is the $k$ of the banks $\left(k_{b}\right)$ and the chain $\left(k_{c}\right)$ in series. For the measured $\Delta k$ the expression becomes, assuming $\Delta k_{c}<k_{b}$,

$$
\Delta k=\frac{k_{b}^{2}}{\left(k_{b}+k_{c}\right)^{2}} \Delta k_{c} \text {. }
$$

From simulations reported in Ref. 13, we can conclude that only the first few atomic layers of the banks deform when pulling on the contact, and therefore the $k$ of the banks is of the same order of magnitude as the $k$ of the chain. Equation (7) shows that in this case the measured $\Delta k$ is only a fraction of $\Delta k_{c}$. The shape of the banks can differ from experiment to experiment, and thus also the $k$ values of the banks. Therefore, the absolute values of the measured $\Delta k$ 's for the chain cannot be determined, only the relative changes. However, it may be possible to determine the stiffness of the banks from an analysis of the evolution of the contact at larger diameters. Further study is underway.

\section{FIRST EXPERIMENTS ON PLATINUM}

Earlier experiments on the forces in atomic structures were only done for gold, which was inherent to the type of instruments used. With the tuning fork MCBJ it is easy to change the material of the wire under investigation. Figure 5 shows the first measurement of force constant and conductance in platinum. It is the same type of measurement as in Fig. 3 and is therefore easy to compare. The general trend is the same in both figures: $k$ decreases with decreasing conductance, and sudden changes in the conductance are accompanied by changes in the force constant. There is, however, a striking difference between the two metals on the conductance plateaus. After an atomic reconfiguration on a conductance plateau (i.e., during elastic elongation), the force constant increases for platinum, whereas for gold it decreases before the rearrangement, except on the chains. This effect will be studied in more detail in a separate publication. ${ }^{30}$ For approximately 50 traces of gold and platinum, we have studied the slope of the change in force constant as a function of 


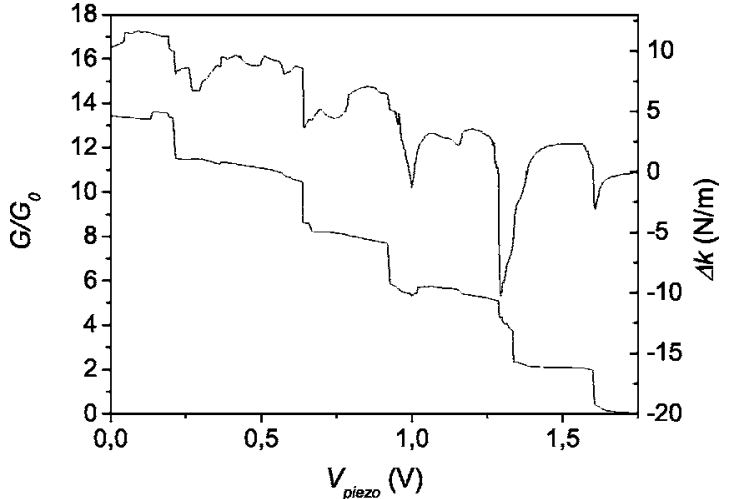

FIG. 5. Measurement of conductance (left axis) and $\Delta k$ (right axis) for platinum as a function of the piezo voltage, which is linear to the elongation of the contact.

conductance. For each trace $\Delta k$ at a certain $V_{\text {piezo }}$ was plotted as a function of the corresponding $\Delta G$. The plot that was obtained with the values of the trace in Fig. 5 is shown in Fig. 6. The inset of Fig. 6 shows a histogram of the values of the slopes $d \Delta k / d g$, with $g=G / G_{0}$. On average, the value of $d \Delta k / d g$ for gold is $30 \%$ higher than for platinum. If we want to compare the change in force constant for gold and platinum per atom, we have to correct the above values with the conductance per atom. Due to the higher number of conductance channels for platinum, the conductance per atom will be higher for platinum; therefore, the change of the force constant per atom will be nearly the same for gold and platinum.

\section{DISCUSSION}

We have shown that a quartz tuning fork resonator can be a versatile instrument for studying the mechanical properties of atomic structures. In comparison with existing instruments it is easier to apply to different metals. We have presented measurements for gold and platinum. The gold measurements are in agreement with the earlier reports. The measurements on platinum show that the absolute value of

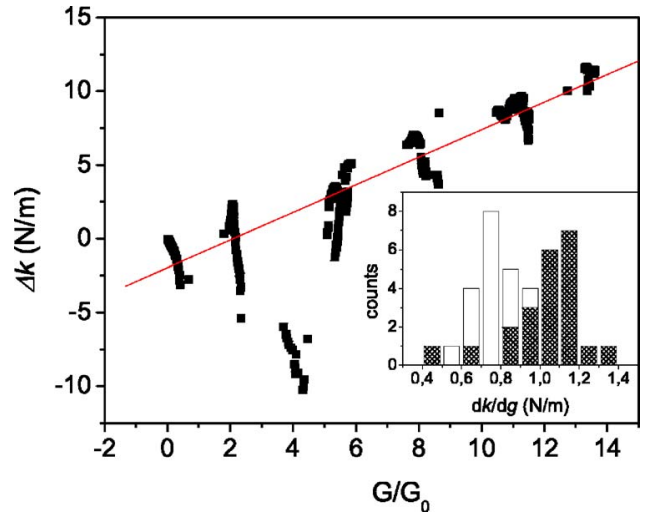

FIG. 6. Plot of $\Delta k$ as a function of $G / G_{0}$ for one trace for a Pt experiment. The line is a linear fit to the data Inset: Histogram of the slope of the change in force constant for gold (filled bars) and platinum (open bars). the changes are of the same order of magnitude as for gold, but the slope of $\Delta k$ on a conductance plateau has a different sign.

\section{ACKNOWLEDGMENTS}

The authors thank F. Giessibl for valuable discussions. This work is part of the research program of the "Stichting FOM," and was partially financed through the SONS program of the European Science Foundation, which is also funded by the European Commission, Sixth Framework Programme, and was also supported by the European Commission TMR Network program DIENOW. C.U. acknowledges the Spanish MEC under Contract No. FIS2004-02356 and the RyC program. T.H.O. acknowledges support from NWO, FOM, and HFSP.

${ }^{1}$ For a review, see N. Agrait, A. Levy Yeyati, and J. M. van Ruitenbeek, Phys. Rep. 377, 81 (2003).

${ }^{2}$ E. Scheer, P. Joyez, D. Esteve, C. Urbina, and M. H. Devoret, Phys. Rev. Lett. 78, 3535 (1997).

${ }^{3}$ E. Scheer, N. Agraït, J. C. Cuevas, A. Levy Yeyati, B. Ludoph, A. MartínRodero, G. Rubio Bollinger, J. M. van Ruitenbeek, and C. Urbina, Nature (London) 394, 154 (1998).

${ }^{4}$ J. K. Gimzewski and R. Möller, Physica B 36, 1284 (1987).

${ }^{5}$ C. J. Muller, J. M. van Ruitenbeek, and L. J. de Jongh, Physica C 191, 485 (1992).

${ }^{6}$ A. I. Yanson, G. Rubio-Bollinger, H. E. van den Brom, N. Agraït, and J. M. van Ruitenbeek, Nature (London) 395, 783 (1998).

${ }^{7}$ A. I. Yanson, I. K. Yanson, and J. M. van Ruitenbeek Nature (London) 400, 1441999.

${ }^{8}$ U. Dürig, J. K. Gimzewski, and D. W. Pohl Phys. Rev. Lett. 57, 2403 (1986).

${ }^{9}$ G. Rubio, N. Agraït, and S. Vieira, Phys. Rev. Lett. 76, 2302 (1996).

${ }^{10}$ S. P. Jarvis, M. A. Lantz, H. Ogiso, H. Tokumoto, and U. Dürig, Appl. Phys. Lett. 75, 3132 (1999).

${ }^{11}$ A. Stalder and U. Dürig, Appl. Phys. Lett. 68, 637 (1996).

${ }^{12}$ G. Rubio-Bollinger, P. Joyez, and N. Agraït, Phys. Rev. Lett. 93, 116803 (2004).

${ }^{13}$ G. Rubio-Bollinger, S. R. Bahn, N. Agraït, K. W. Jacobsen, and S. Vieira, Phys. Rev. Lett. 87, 026101 (2001).

${ }^{14}$ M. R. Sorensen, M. Brandbyge, and K. W. Jacobsen, Phys. Rev. B 57, 3283 (1998)

${ }^{15}$ F. J. Giessibl, Appl. Phys. Lett. 73, 3956 (1998).

${ }^{16}$ F. J. Giessibl, Appl. Phys. Lett. 76, 1470 (2000).

${ }^{17}$ W. H. J. Rensen, N. F. van Hulst, A. G. T. Ruiter, and P. E. West, Appl. Phys. Lett. 75, 1640 (1999).

${ }^{18}$ J. Rychen, T. Ihn, P. Studerus, A. Herrmann, and K. Ensslin, Rev. Sci. Instrum. 70, 2767 (1999).

${ }^{19}$ H. Edwards, L. Taylor, W. Duncan, and A. J. Melmed J. Appl. Phys. 82, 980 (1997).

${ }^{20}$ K. Karrai and R. D. Grober, Proc. SPIE 2535, 69 (1995).

${ }^{21}$ W. A. Atia and C. C. Davis, Appl. Phys. Lett. 70, 405 (1997).

${ }^{22}$ F. J. Giessibl, S. Hembacher, H. Bielefeldt, and J. Mannhart, Science 289, 422 (2000).

${ }^{23}$ Supplier: Farnell InOne, Nautilusweg 39, 3542 AT Utrecht, the Netherlands. Manufacturer: AEL CRYSTALS nr:4811-32 K768.

${ }^{24}$ R. D. Grober, J. Acimovic, J. Schuck, D. Hessman, P. J. Kindlemann, J. Hespanha, A. S. Morse, K. Karrai, I. Tiemann, and S. Manus, Rev. Sci. Instrum. 71, 2776 (2000).

${ }^{25}$ LABVIEW 6i, National Instruments, Austin, TX.

${ }^{26}$ C. Schönenberger and S. F. Alvarado, Rev. Sci. Instrum. 60, 3131 (1989).

${ }^{27}$ C. Untiedt, A. I. Yanson, R. Grande, G. Rubio-Bollinger, N. Agraït, S. Vieira, and J. M. van Ruitenbeek, Phys. Rev. B 66, 235416 (2002).

${ }^{28}$ B. Ludoph, M. H. Devoret, D. Esteve, C. Urbina, and J. M. van Ruitenbeek, Phys. Rev. Lett. 82, 1530 (1999).

${ }^{29}$ B. Ludoph and J. M. van Ruitenbeek, Phys. Rev. B 59, 12290 (1999).

${ }^{30}$ A. M. C. Valkering, A. Mares, and J. M. van Ruitenbeek (unpublished). 